Check for updates

Cite this: RSC Adv., 2017, 7, 21686

Received 23rd February 2017

Accepted 12th April 2017

DOI: $10.1039 / c 7 r a 02235 b$

rsc.li/rsc-advances

\title{
Anisotropic polydopamine capsules with an ellipsoidal shape that can tolerate harsh conditions: efficient adsorbents for organic dyes and precursors for ellipsoidal hollow carbon particles $\uparrow$
}

\author{
Lu Yang, $\ddagger^{\mathrm{a}}$ Cong Wang,,$^{\mathrm{a}}$ Zihan Ye, ${ }^{\mathrm{b}}$ Pengjiao Zhang, ${ }^{\mathrm{b}}$ Songhai Wu, ${ }^{\text {a }}$ Shaoyi Jia, ${ }^{\mathrm{a}}$ \\ Zhanyong $\mathrm{Li}^{\mathrm{b}}$ and Zhenkun Zhang (D)*b
}

\begin{abstract}
Polymeric capsules often buckle, collapse or even break when being processed in the dried state into other materials under high temperature and pressure due to moderate mechanical rigidity. In the case of nonspherical capsules, to keep their precious anisotropic morphology intact under harsh conditions is even more challenging since the whole surface of such kinds of capsules does not experience the same stress or strain due to the different surface curvatures. In the current work, we reported a strategy to prepare polydopamine (PDA) capsules with an ellipsoidal shape and enhanced mechanical rigidity using polystyrene ellipsoids as the sacrificial anisotropic templates. Bio-inspired oxidation induced selfpolymerization of dopamine can form conformal PDA coatings on polystyrene ellipsoids of various aspect ratios and sizes. Several strategies have been exploited to increase the thickness of the PDA shell, among which, iterating PDA coating produces ellipsoidal PDA capsules with a thick and robust shell. These ellipsoidal PDA capsules can survive carbonization at temperatures as high as $800{ }^{\circ} \mathrm{C}$ and were directly turned into $\mathrm{N}$-doped carbon capsules with a well-defined ellipsoidal shape, excluding the necessity of removing the sacrificial templates after carbonation. Furthermore, the rigid PDA ellipsoidal capsules are efficient adsorbents for organic dyes in contaminated water and have impressive adsorption efficiencies as high as $200 \mathrm{mg} \mathrm{g}^{-1}$.
\end{abstract}

\section{Introduction}

Inspired by the key biomaterial that is responsive for mussels' amazing capability of adhering to any surface, polydopamine (PDA) derived from oxidation induced self-polymerization of dopamine under basic $\mathrm{pH}$ conditions has recently become popular due to its many potential applications. ${ }^{1,2}$ PDA can coat the surface of many inorganic, organic and biological materials of various dimensions, offering a convenient means for surface modifications..$^{1-3}$ The morphology of a new material is one of the critical material parameters that determines its end applications. In this regard, capsules of PDA at a nano or micro scale have also attracted wide interest with the hope to extend this material into such diverse fields as drug delivery, catalysis,

${ }^{a}$ School of Chemical Engineering and Technology, Tianjin University, Tianjin 300072, China. E-mail:wusonghai@tju.edu.cn

${ }^{b}$ Key Laboratory of Functional Polymer Materials of Ministry of Education, Institute of Polymer Chemistry, College of Chemistry, Nankai University, Tianjin 300071, China. E-mail:zkzhang@nankai.edu.cn

$\dagger$ Electronic supplementary information (ESI) available: List of typical absorbents for MB, more XPS, SEM and TEM photos of PDA coated ellipsoids and capsules. See DOI: $10.1039 / \mathrm{c} 7 \mathrm{ra02235b}$

$\ddagger$ These authors contributed equally to this work. energy, etc. ${ }^{3-7}$ PDA and other polymeric capsules are mainly prepared based on templates of soft or hard particles, followed by multi-step etching under harsh conditions. ${ }^{5,6,8-12}$ It is noted that most of PDA capsules prepared so far have a spherical shape ${ }^{4-7,11}$ while non-spherical ones may have additional advantages in cargo loading, cell uptake and enhanced packing efficiency in energetic materials. ${ }^{13-15}$ However, preparation of non-spherical polymeric capsules is generally challenging. ${ }^{16,17}$ First of all, it is difficult to form a uniform and conformal coating around the non-spherical particulate templates that have surfaces with varied curvatures. ${ }^{16,18,19}$ Furthermore, the non-spherical shape of the capsules often lose after removing the templates due to high residual stresses or osmotic pressures. ${ }^{16,18,19}$ In terms of such challenges, PDA coating is valuable due to its capability to form a conformal coating layer on particulate templates with any kinds of shapes. ${ }^{5,6,9-12}$ Even so, PDA capsules with an anisotropic shape have barely been reported. ${ }^{20}$

Besides the shape of the polymeric capsules, the rigidity or mechanical stability, especially, the ability to keep the shape intact under some extreme conditions is one of the key traits of hollow capsules for many practical applications. ${ }^{21}$ When suspended in solvent, polymeric capsules can keep its original 
morphology in most of cases. However, many capsules will buckle, collapse or even break when being subjected to drying, high vacuum as in the case of electronic microscope or experiencing external forces such as exerted by the cantilever of AFM..$^{\mathbf{4}, \mathbf{6 , 7 , 9 , 1 0 , 2 2 - 2 5}}$ Furthermore, polymeric capsules often need to be processed into other materials in the dried state and under high temperature and pressure. ${ }^{\mathbf{1 5 , 2 6 , 2 7}}$ Keeping the intactness of their precious capsule-like shape is demanding during such stringent processes since the loss of the shape unavoidably devalues the advantages brought by the hollow cavity, and high specific surface area. For instance, highly charged spherical polystyrene capsules were dried into three dimensional colloidal crystals that can be used as photonic crystals for chemical sensing by the change of the structure color. ${ }^{28}$ However, buckling of the polystyrene capsules inside the dried crystals introduced defects and inhomogeneities that severely decrease the quality of the photonic crystals. In self-healing polymers realized by capsule based strategies, breaking of the capsules at high processing temperature $\left(>100{ }^{\circ} \mathrm{C}\right)$ can cause premature leaking of the core to the host matrix, leading to reduced self-healing capability. ${ }^{29}$ In addition, calcinations at very high temperature more than $800{ }^{\circ} \mathrm{C}$ and the dried state is a common way to turn organic carbon precursors into carbon materials. $^{30-34}$ It is highly challenging, if not impossible, to directly burn polymeric capsules into carbon capsules without losing their original morphology. ${ }^{26}$ In the case of non-spherical capsules, to keep the shape intact under afore-mentioned harsh conditions is even more elusive since the whole surface does not experience the same stress or strain due to the different surface curvatures. ${ }^{18}$ Therefore, enhancing the mechanical robustness of polymeric capsules under harsh conditions has attracted wide interest in the field of polymeric capsules. A very recent example of such efforts is the very tough polymeric polyhedral capsules reported by Caruso. ${ }^{35}$

In the above context, spherical PDA capsules have been demonstrated to have higher mechanical rigidity than other polymeric capsules that are prepared via layer-by-layer assembly, seeded polymerization, etc. ${ }^{36}$ This can be ascribed to the rigid backbone formed by oxidation-induced selfpolymerization of dopamine and highly crosslinked chains. Even so, PDA capsules are often in the bulked or collapsed state after airdrying, indicating a moderate mechanical

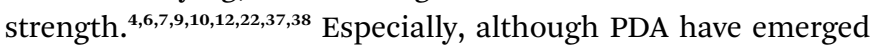
as a very attractive precursor for nitrogen-doped carbon materials, preparation of hollow carbon particles or carbon capsules by carbonization of PDA involves multiple steps including PDA coating of hard templates, carbonization and template removing. ${ }^{30,31,33,34}$ Carbonization of PDA coated templates might lead to the contamination of the carbon materials by the underlying templating materials, which can be avoid by direct carbonization of pure PDA capsules into carbon capsules. However, such strategy has barely been exploited since PDA capsules might not survive the high temperature $\left(>800{ }^{\circ} \mathrm{C}\right)$ of carbonization. In addition, most of carbon capsules derived from PDA have a spherical shape, only a few examples of nonspherical one have been prepared via templating on hard inorganic particles and acid etching after the carbonation. ${ }^{39}$

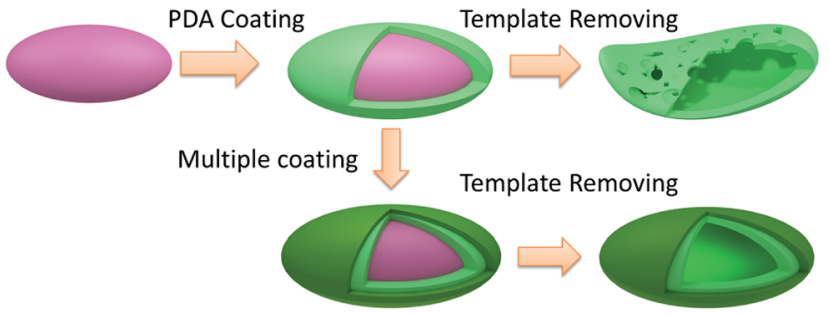

Scheme 1 Schematic illustration of preparing PDA capsules with an ellipsoidal shape and enhanced mechanical rigidity by templating on polystyrene ellipsoids.

Besides precursors for carbon materials, PDA based materials have also exhibited excellent adsorption of metal ions or organic dyes, which have been exploited in water purification. ${ }^{\mathbf{4 0}-42}$ PDA capsules are expected to further enhance such adsorption efficiency due to their double interfaces, high specific surface area. However, the moderate mechanical strength of PDA capsules delays their practical applications in water purification in the form of dried powders or packing into columns under high pressure for chromatograph. Due to its different surface curvatures that experience different stress or strain, non-spherical PDA capsules are expected to have much weaker mechanical strength than their spherical counterparts, which make direct carbonation into carbon capsules with an anisotropic shape or drying into powders as adsorbents are highly challenging.

In the current contribution, we report a strategy to prepare PDA capsules with an ellipsoidal shape and enhanced mechanical rigidity by templating on polystyrene ellipsoids of various aspect ratio and sizes (Scheme 1). By removing the PS template we can prepare ellipsoidal PDA capsules with a size ranging from micro to nanometer. While keeping their shape in solution, the ellipsoidal PDA capsules obtained from the standard one-pot PDA coating buckle, collapse and break when being subjected to air-drying. Direct carbonization at $800{ }^{\circ} \mathrm{C}$ resulted in fragmentation of the PDA ellipsoidal capsules. Several strategies have therefore been exploited to control the thickness of the PDA shell with the hope to increase their mechanical rigidity of the shell, among which, iterating PDA coating gives rise to ellipsoidal PDA capsules with a thick shell without contamination of pure PDA spheres. These ellipsoidal capsules can survive the heating under high temperature and were directly turned into N-doped carbon capsules with a well-defined ellipsoidal shape. Furthermore, we shall also demonstrate that such stable PDA ellipsoidal capsules are efficient adsorbents for organic dyes in contaminated water. An impressive high adsorption efficiency of $200 \mathrm{mg} \mathrm{g}^{-1}$ was achieved.

\section{Experimental}

\subsection{Material}

Three kinds of polystyrene ellipsoids were prepared as detailed in our previous work: ${ }^{43}$ one with a long and short axis of $c a .6$ and $0.7 \mu \mathrm{m}$, one with a long and short axis of $c a .3$ and $1 \mu \mathrm{m}$ and one with a long and short axis of 600 and $300 \mathrm{~nm}$, respectively. 
Dopamine hydrochloride (DA), fluoresceinamine isomer I, and Tris(hydroxymethyl)amino methane (Tris) were purchased from Sigma-Aldrich. All other materials were obtained from J\&K (Beijing, China).

\subsection{Preparation of ellipsoidal polydopamine capsules}

PDA coating of the polystyrene ellipsoid has been detailed in our previous work..$^{43} \mathrm{In}$ brief, to $10 \mathrm{~mL}$ suspension of PS ellipsoids of $10 \mathrm{mg} \mathrm{mL} \mathrm{mL}^{-1}$ in Tris-HCl buffer $(\mathrm{pH}=8.5,10 \mathrm{mM})$, dopamine was directly added under strong stirring to a final concentration of $0.1 \mathrm{mg} \mathrm{mL} \mathrm{m}^{-1}$. The coating reaction was carried out for 10 hours under stirring that is enough to prevent the sedimentation of the ellipsoids. After coating, three rounds of centrifuge and redispersing in ethanol were performed to purify the PDA coated particles (PS@PDA). To prepare ellipsoidal PDA capsules, $1 \mathrm{~mL}$ of PS@PDA in ethanol were centrifuged to remove solvents. To the sediments, $4 \mathrm{~mL}$ of tetrahydrofuran (THF) was added. The resulted suspension was stirred for 5 hours. The PDA capsules were collected by low-speed centrifuge (2000g) and redispersed in THF for 5 hours. This procedure was repeated three times to make sure all of the PS templates are removed completely. The asprepared capsules was finally redispered in Tris- $\mathrm{HCl}$ buffer $(\mathrm{pH}$ $=8.5,10 \mathrm{mM}$ ) for further characterizations.

To investigate the influence of the dopamine concentration on the thickness of the PDA shell, final dopamine concentrations of 1 and $2 \mathrm{mg} \mathrm{mL}^{-1}$ were achieved by adding various amount of dopamine to the PS ellipsoidal suspension in Tris-HCl buffer to obtain while the coating time was kept at 10 hours. Similarly, while the concentration of dopamine was fixed at $0.1 \mathrm{mg} \mathrm{mL}^{-1}$, aliquots of the reaction mixture were taken at 15 and 25 hours and purified to investigate the influence of the coating time.

\subsection{Iterating PDA coating to prepare ellipsoidal polydopamine capsules with varied shell thickness}

The PS@PDA hybrid particles based on micrometer-sized ellipsoids with an aspect ratio of 3 were used for iterating PDA coating. The PS@PDA obtained under the default coating condition $\left(C_{\text {dopame }}=0.1 \mathrm{mg} \mathrm{mL}^{-1}\right.$ and coating time $=10$ hours $)$ were purified and resuspended in Tris- $\mathrm{HCl}$ buffer $(\mathrm{pH}=8.5,10$ $\mathrm{mM}$ ). To the suspension, dopamine was added to achieve concentration of $0.1 \mathrm{mg} \mathrm{mL} \mathrm{m}^{-1}$ and the coating reaction was performed for another 10 hours under constant agitating. This procedure was further repeated on the same PS@PDA hybrid particles for each specific coating cycle. To remove the PS core, the PS@PDA hybrid particles were suspended in THF for at least 25 hours during which THF was replaced with fresh THF for three times.

\subsection{Fluorescent dye labeling of the PDA capsules}

The micrometer-sized PDA capsules with an aspect ratio of 3 and various shell thickness were used for the dye labeling. PDA capsules in $2 \mathrm{~mL}$ phosphate buffer $(10 \mathrm{mM}, \mathrm{pH}=8.2)$ with a concentration of $10 \mathrm{mg} \mathrm{mL} \mathrm{m}^{-1}$ were first prepared. To the suspension, fluoresceinamine was added to achieve a dye concentration of $10 \mu \mathrm{g} \mathrm{mL}{ }^{-1}$. The resulted mixture was stood for 24 hour in dark under constant stirring. After this step, the reaction mixture was dialyzed against water to remove unreacted dyes. The capsules were collected and further washed in distilled water by centrifuge and redispersion.

\subsection{Carbonization of PDA capsules to prepare $\mathrm{N}$-doped carbon capsules with an ellipsoidal shape}

The micrometer-sized PDA capsules with an aspect ratio of 3 obtained from one and four cycles of PDA coating was tested for direct carbonation under $\mathrm{N}_{2}$ atmosphere. The capsules were first carbonized at $400{ }^{\circ} \mathrm{C}$ for $2 \mathrm{~h}$ with a heating rate of $1{ }^{\circ} \mathrm{C}$ $\min ^{-1}$, and then further treated at $800{ }^{\circ} \mathrm{C}$ for $3 \mathrm{~h}$ with a heating rate of $5{ }^{\circ} \mathrm{C} \mathrm{min}^{-1}$. The carbonization product was characterized directly without further treatment.

\subsection{Adsorption of methylene blue (MB) from water}

The micrometer-sized PDA capsules with an aspect ratio of 3 obtained from four cycles of PDA coating were tested for adsorption of methylene blue (MB). The adsorption experiments were performed follow the exact procedure as outlined in ref. 44 in order to compare with the result of pure PDA particles. To $2 \mathrm{~mL}$ of $50 \mathrm{mg} \mathrm{L}^{-1} \mathrm{MB}$ solution at $\mathrm{pH} 6.5$ was added $0.2 \mathrm{mg}$ PDA capsules. The container was shaken on a shaking incubator at a speed of $150 \mathrm{rpm}$. The concentrations of MB left in supernatant were determined using a UV-vis spectrophotometer at preset $10 \mathrm{~min}$ interval in the range of $120 \mathrm{~min}$. The amount of MB adsorbed per unit mass of PDA capsules $(q)$ and the dye removal efficiency $(R)$ were calculated according to the following eqn (1) and (2), respectively ${ }^{32}$

$$
\begin{gathered}
q=V\left(C_{0}-C_{\mathrm{t}}\right) / m \\
R=100\left(C_{0}-C_{\mathrm{t}}\right) / C_{0}
\end{gathered}
$$

where $m(\mathrm{~g})$ is the mass of PDA capsules and $V(\mathrm{~L})$ is the volume of MB solution. $C_{0}$ and $C_{\mathrm{t}}\left(\mathrm{mg} \mathrm{L}^{-1}\right)$ are the initial and final (after adsorption) concentration of MB solution, respectively.

\subsection{Characterization}

Scanning (SEM) and transmission (TEM) electron microscope were performed with a JSM-7500F SEM and JEM-100CXII TEM (JEOL, Japan), respectively. Samples for SEM were prepared by putting a diluted suspension of particles in ethanol to a clean silicon wafers and drying in air at room temperature. Samples for TEM were prepared by placing a drop of the particle suspension ethanol on a carbon-coated copper grid, and then evaporating at ambient temperature. X-ray photoelectron spectroscopy (XPS) analysis of the hybrid particles and PDA capsules was conducted on a GENESIS 60S X-ray photoelectron spectroscope (EDAX, USA). Thermogravimetric analysis (TGA) was performed on a TG 209 F3 (Netzsch, Germany) under $\mathrm{N}_{2}$ atmosphere and in the temperature range from 50 to $800{ }^{\circ} \mathrm{C}$. The fluorescent images of the fluorescently labeled ellipsoids were recorded on a Zeiss Z2 fluorescent microscopy (Zeiss, Germany). Differential interference contrast (DIC) microscopy was performed on an Olympus BX 51 microscopy with DIC optics. 


\section{Results and discussion}

\subsection{Preparation of PDA capsules with an ellipsoidal shape}

PDA coating of nano- or colloidal particles of various kinds of

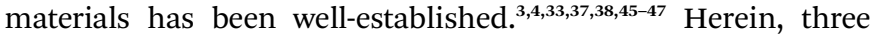
kinds of PS ellipsoids prepared from a well-known thermostretching method were used as the templates: ${ }^{48}$ two kinds of micrometer-sized ellipsoids with different aspect ratios (ARs) prepared from the same PS microsphere and one kind of nanoscale ellipsoids. In our previous work, we have optimized the PDA coating condition that leads to PDA coated colloidal particles without contamination of the pure PDA side products. ${ }^{43}$ The same coating procedure was used in the current work to coat the PS ellipsoids with PDA. For this, dopamine was added to the ellipsoid suspension in Tirs-HCl buffer and stirred for certain time. After washing by centrifuge and re-dispersing in water, the PDA coated PS ellipsoids (PS@PDA) was incubated in THF to remove the PS core. The PS core is not crosslinked and therefore the linear PS chains dissolved in THF can diffuse out of the PDA shell, as confirmed by many previous works. ${ }^{6,23,24,40,49}$ TEM and SEM of the PDA coated particles and the ones after removing PS are listed in Fig. 1. Mass loss profiles of the capsules from the thermogravimetric analysis (TGA) are similar to that of pure PDA (Fig. 2A), confirming that the PS templates have been removed. The PDA coated particles and capsules were further characterized by X-ray photoelectron spectroscopy (XPS) (Fig. S1 in ESI $\dagger$ ). The C 1s high-resolution spectrum of the XPS survey of the PDA capsules can be deconvoluted into several sub-peaks with values that correspond to the functional groups of intermediate compounds derived from dopamine (Fig. 2B): $284.98 \mathrm{eV}(\mathrm{C}=\mathrm{C}$, $\mathrm{C}-\mathrm{C}$, and $\mathrm{C}-\mathrm{H}), 286.24 \mathrm{eV}(\mathrm{C}-\mathrm{N}, \mathrm{C}=\mathrm{N}$, and $\mathrm{C}-\mathrm{OH})$, and $289.04 \mathrm{eV}$ $(\mathrm{C}=\mathrm{O}$ of the quinone). Similarly, the $\mathrm{O} 1$ s core-level spectrum can be fitted into two typical peaks at $532.39 \mathrm{eV}(\mathrm{C}=\mathrm{O}$ of quinone) and $533.36 \mathrm{eV}$ (C-OH of catechol) (Fig. 2C). ${ }^{46,50}$ In addition, compared to the PS template, significant intensity of nitrogen is also detected by XPS in the case of the PDA capsules (Fig. 2D).

TEM and SEM revealed that the PDA capsule structure maintains the general ellipsoidal shape and accurately replicate the size and aspect ratio of the respective templates, leading to microscale ellipsoidal PDA capsules with an aspect ratio of 6 and 3 as well as a nanoscale one with aspect ratio of 2 (Fig. 1). Especially, the highly curved surface at the tips of the ellipsoidal templates were preserved: capsules from the micrometer-sized ellipsoids with an $\mathrm{AR}=6$ have sharper tips than the ones from particles with an AR of 3 . Under the normal coating conditions ( $0.1 \mathrm{mg} \mathrm{mL}^{-1}$ of dopamine and coating time of $10 \mathrm{~h}$ ), the shell thickness of the capsules is around $20 \mathrm{~nm}$. We also tested PDA coating of the micrometer-sized ellipsoids with an AR of 6 using a very low dopamine concentration $(0.04 \mathrm{mg}$ $\mathrm{mL}^{-1}$ ), and capsules with a very thin PDA layer but still an ellipsoidal shape were obtained (Fig. S2 in ESI $\dagger$ ). These results suggest that PDA coating can form a conformal layer on anisotropic particles, regardless of the size and surface curvature. ${ }^{20,51}$ After removing the templates, the resulted PDA shell has the capability to remain the general shape of the templates due to the inherent rigidity of PDA. ${ }^{36,52}$ In the rest of this work, we mainly focus on PDA capsules from the micrometer-sized ellipsoid with an $\mathrm{AR}=3$ unless otherwise noted.

\subsection{Morphology of ellipsoidal PDA capsules in the suspended and dried state}

The PDA capsules that have never been in the dried state were checked with differential interference contrast (DIC) and
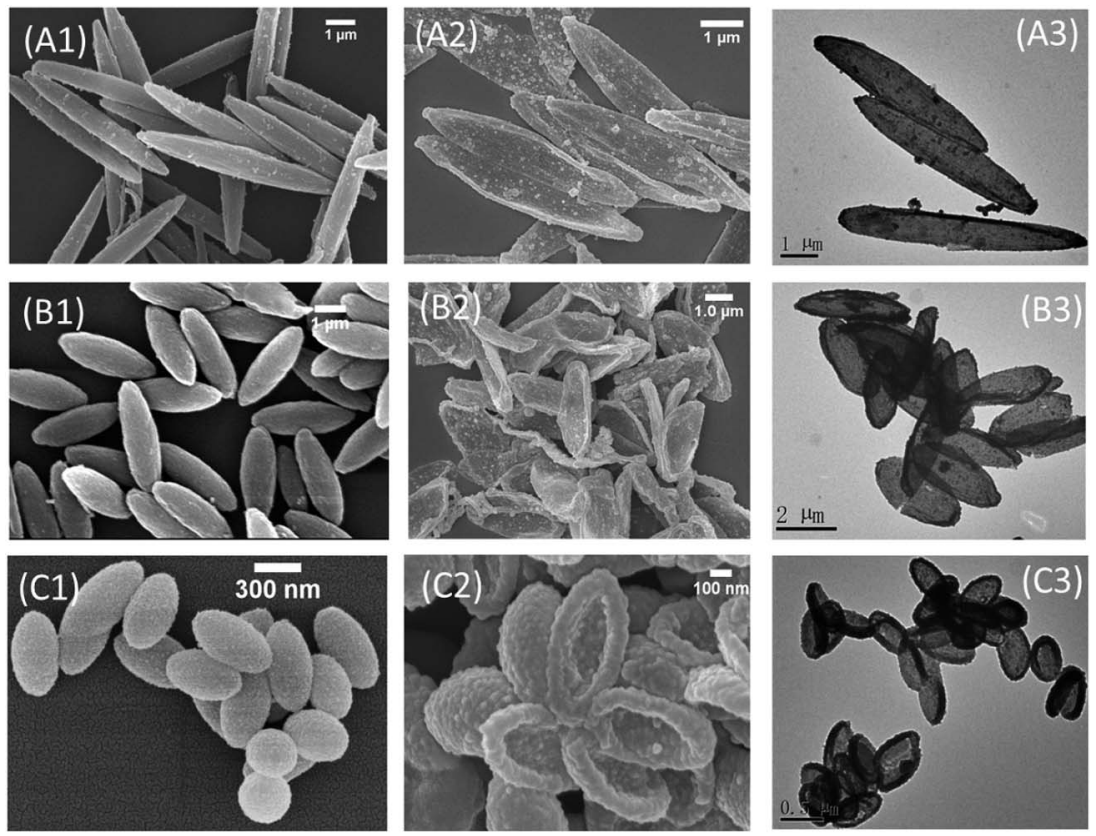

Fig. 1 TEM and SEM of PDA coated PS ellipsoids (left column) and ellipsoidal PDA capsules (middle and right column). (A) and (B) are PDA coated micrometer-sized PS ellipsoids and capsules with an aspect ratio of 6 and 3, respectively. (C) Nanoscale ellipsoids. The coating condition is as follows: $C_{\text {dopamine }}=0.1 \mathrm{mg} \mathrm{mL}^{-1}$ and the coating time is 10 hours. 

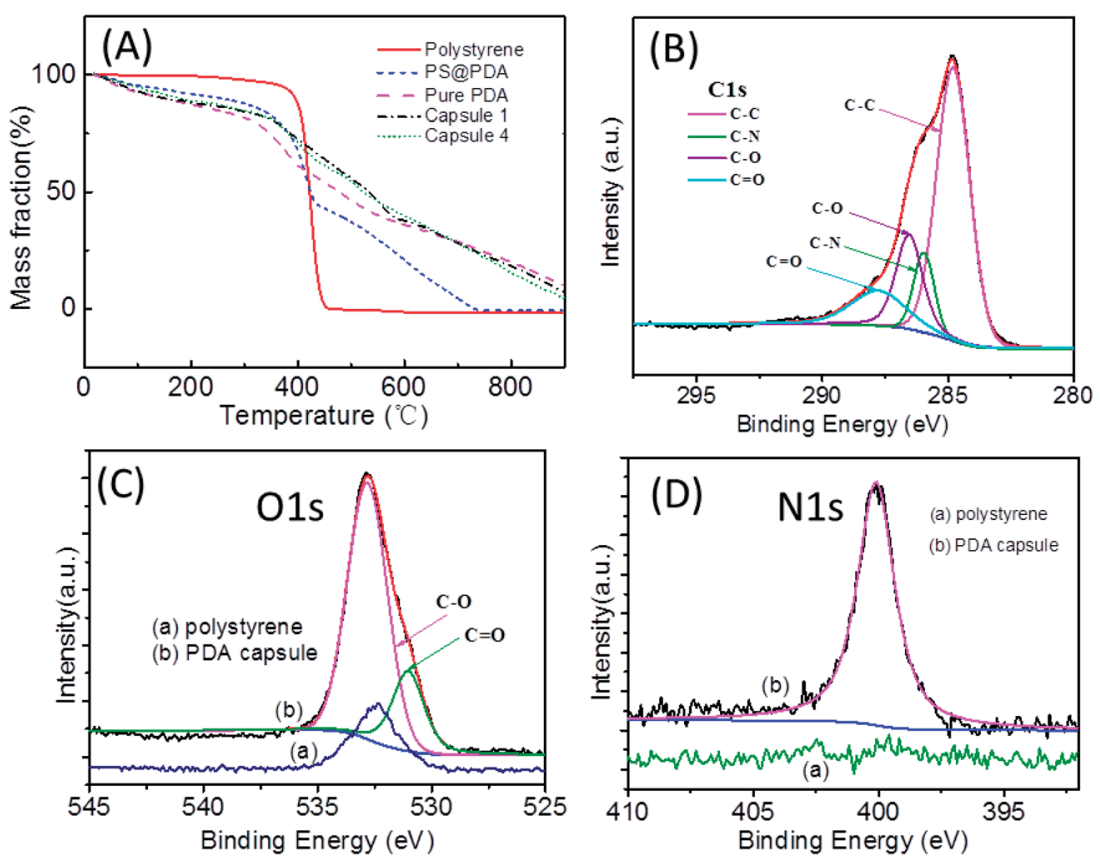

Fig. 2 TGA (A) and XPS analysis (B-D) of the PDA coated PS ellipsoids and the resulted capsules. The PDA coated ellipsoids and capsules are based on the micrometer-sized ellipsoids with an aspect ratio of 3 . Capsule 1 and 4 in (A) refer to PDA capsules that are obtained from one and four cycles of iterating PDA coating, respectively.

fluorescent microscopy to reveal the morphology of ellipsoidal PDA capsules in the suspended state (Fig. 3 and S3 in ESI $\dagger$ ), where both the interior cavity and the outer side of the capsules are filled with the same solvent like water. DIC of the capsules reveals an intact ellipsoidal morphology (inset of Fig. 1B and S3 in ESI $\dagger$ ). In addition, the capsules can be conveniently labeled with fluorescent dyes due to the rich chemistry of PDA. Fluorescent microscopy images of the FITC labeled capsules and the parent PS@PDA core-shell particles without removing the PS core are listed in Fig. 3A and B, together with quantitative analysis of the intensity alone the long and short axis of the particles. Compared to the fluorescent PS@PDA ellipsoids, the PDA capsules are slightly swollen but still have a well-defined ellipsoidal morphology without any buckling (Fig. 3A and B). These results confirm that dissolution and diffusion of the PS chains do not disrupt the capsules. The fluorescent intensity was further analyzed by plot profiles along the long and short axis of the respective particles (Fig. 3C and D). Interestingly, while the PS@PDA ellipsoid consists of a fluorescent halo and a dark core, fluorescent signals also exist in the cavity of the fluorescent PDA capsules, suggesting that fluorescent molecules can diffuse inside the capsules and label the interior PDA surface. ${ }^{53}$ This is a very important property that benefits the dye adsorption as will be discussed later.

Both DIC and fluorescent microscopy indicate that PDA capsules from the above one-pot PDA coating can keep the ellipsoidal morphology when suspended in solvent. However, TEM and SEM of dried capsules reveal a buckled and collapsed morphology and some capsules are broken, regardless of the aspect ratio and size of the capsules (Fig. 1). This is consistent with previous results of spherical PDA capsules
(Fig. S4 $\dagger)^{4,6,7,9,12,24,37,38,40}$ Interestingly, although collapsing of the spherical PDA capsules isotopically occurs to all directions around the curved surface (Fig. S4 in ESI $\dagger$ ), ${ }^{9,38}$ the micrometersized ellipsoidal PDA capsules collapse along their side of the long axis into flattered shells. ${ }^{54}$ In a similar way, the nanosized PDA capsules buckle into the bowel-like structure with an ellipsoids shape (Fig. 1C). Previous works have indicated that strain/stress in homogenously distribute along the curved surface, vertices and edges of the non-spherical capsules. ${ }^{18,55}$ The latter two are rigid and provide mechanical reinforcement to restrict the buckling behavior of non-spherical capsules. Therefore, in the case of ellipsoidal capsules, air-drying induced stress mainly release in homogeneously through the mechanically weak side walls. ${ }^{56}$ Since the capsules are intact in solvent (Fig. 3), the collapsed and broken ellipsoidal PDA capsules must be due to the stress of air drying. As will be shown later, heating the ellipsoidal PDA in the dried state under high temperature $\left(>400{ }^{\circ} \mathrm{C}\right)$ can further disintegrate them into fragments. Therefore, the inherent rigidity of PDA can maintain the anisotropic morphology of the template but is not strong enough to keep the integrity of the capsules under dying and other harsh conditions.

\subsection{Ellipsoidal PDA capsules with enhanced mechanical rigidity via iterating PDA coating}

Naturally, the simple way to enhance the mechanical rigidity of the ellipsoidal PDA capsules is to increase the thickness of the PDA shell. ${ }^{4,6,7}$ For this, two possibilities can be exploited: one is to vary the initial PDA concentrations $\left(C_{\mathrm{dp}}\right)^{4,6,22}$ and another is to prolong the PDA coating time., ${ }^{7,22}$ However, increasing the 

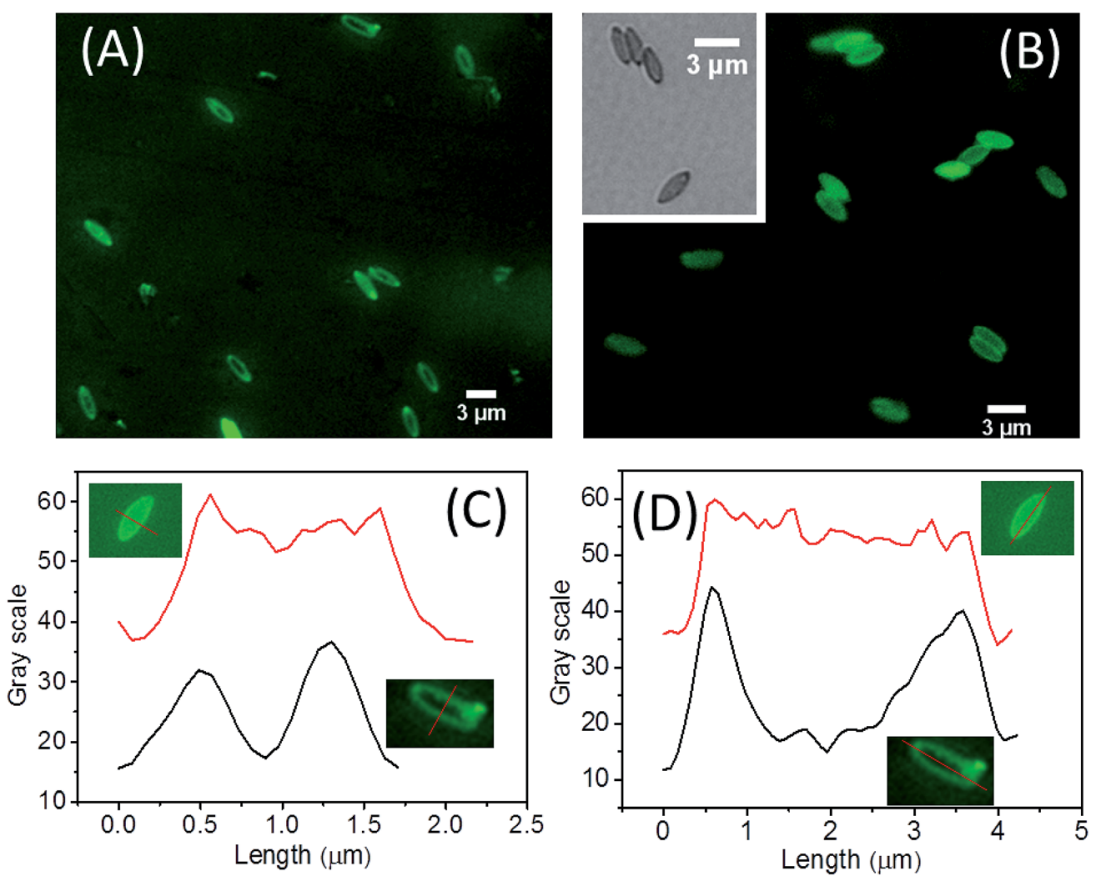

Fig. 3 DIC and fluorescent microscopy of PDA coated PS ellipsoids (A) and PDA capsules (B). The panels of (C) and (D) are the plot profiles along the long and short axis of the PDA coated particles (black curves) and PDA capsules (red curves). The PDA coated ellipsoids and capsules are based on the micrometer-sized ellipsoids with an aspect ratio of 3.

dopamine concentration can lead to a large amount of pure PDA particles of several hundreds of $\mathrm{nm}$ that are difficult to be separated from the main product, especially in the case of coating nanoscale ellipsoids (Fig. S6 in ESI†). In addition, the thickness of the PDA shells only slightly increases by prolonging the coating time (Fig. S5 in ESI†). ${ }^{7}$ This is due to the unique selfpolymerization chemistry of dopamine as we discussed before. ${ }^{43}$ Generally, dopamine is oxidized into some polymerizable species at the early stage of PDA coating, some of which adsorb onto the template surface while some continue selfpolymerizing into non-soluble aggregates in bulk. The adsorbed polymerizable species on the particle surface condense into a conformal layer, onto which PDA aggregates in the form of particles of various sizes can further deposit. Therefore, the PDA capsules often consist of a relatively smooth layer decorated with nanoscale PDA particles (Fig. 2A). These two procedures compete with each other. Initial high concentration of dopamine leads to very concentrated polymerizable species that accelerate the self-polymerization and condensation of the species into pure PDA particles that precipitate out. In addition, at a specific dopamine concentration, the thickness increasing of the PDA layer levels off after several hours due to depletion of oxygen and/or dopamine.

Therefore we explored the strategy of iterating coating with the optimal dopamine concentration of $0.1 \mathrm{mg} \mathrm{mL}^{-1}$, which we have found give the best coating while producing negligible side products of pure PDA particles. ${ }^{6,9,38}$ After each coating, the PDA coated ellipsoids was washed with Tris- $\mathrm{HCl}$ buffer and then suspended into a freshly prepared dopamine solution of $0.1 \mathrm{mg}$ $\mathrm{mL}^{-1}$. In this way, we can increase the thickness of the PDA without producing pronounced pure PDA side productions (Fig. 4). Indeed, after the second round of coating, the amount of ellipsoidal capsules with an intact ellipsoidal shape that survived the drying for electron microscopy analysis increases dramatically (Fig. 4A and 5A). After the fourth cycle of PDA coating, most of the capsules have an intact ellipsoidal shape (Fig. 4C and S7 in ESI†े). With increasing cycles of PDA coating, the thickness of the PDA capsules also increases (Fig. 5B). The surface of the capsules becomes rougher and rougher (Fig. 4). TGA analysis of the densely coated PDA capsules from the fourth cycle of coating have a mass loss profile similar to that of the pure PDA, suggesting that the PS core are removed by THF (Fig. 2A). The hollow cavity of the PDA capsules after the fourth cycle of coating could not be revealed by both TEM and SEM due to the thick PDA shell with a thickness of $c a$. $65 \mathrm{~nm}$ (Fig. 4C), as also reported by others. ${ }^{6,9,38}$ TEM and SEM of the capsules after mechanically crashing clearly reveal the capsule structure (insets of Fig. 4C). In addition, DIC and fluorescent microscopy also confirm the capsule-like structure (Fig. S3 in ESI†).

\subsection{Preparation of N-doped ellipsoidal carbon capsules}

PDA based materials have recently been demonstrated as excellent precursors for $\mathrm{N}$-doped carbon materials. ${ }^{30}$ Combining with the versatile PDA coating of nano- and colloidal particles, a plethora of hollow carbon particles or carbon capsules have been conveniently prepared and have found many applications in water purification, catalysis, advanced battery technologies. ${ }^{30,33,34}$ However, most carbon capsules derived from PDA have a spherical shape and are prepared in multiple steps that involve PDA coating, 

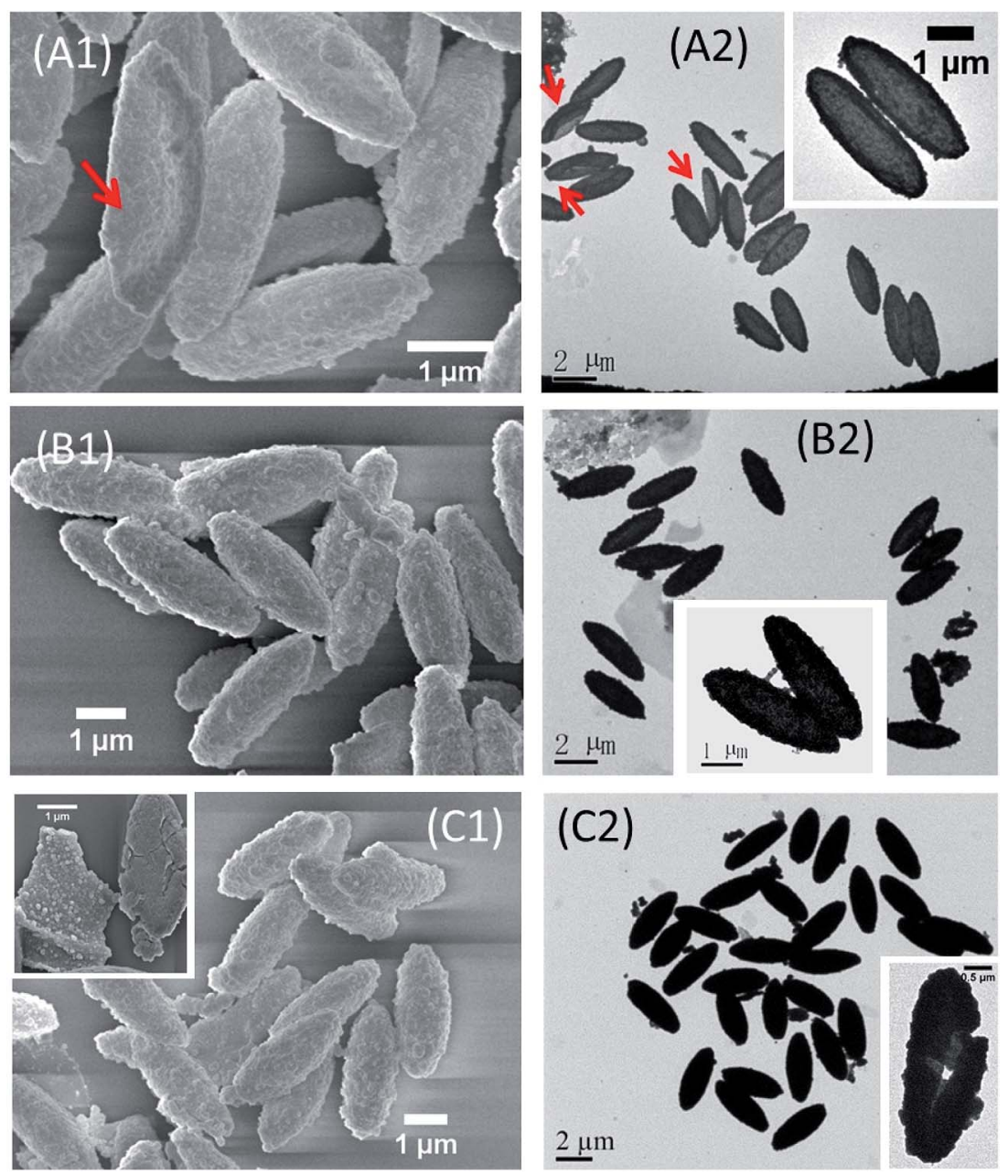

Fig. 4 PDA capsules with increasing shell thickness and mechanical rigidity by iterating PDA coating. SEM and TEM images in (A), (B) and (C) are two, three and four cycles of PDA coating, respectively. Arrows in (A) highlight buckled and broken PDA capsules. Insets in C1 and C2 are the capsules after mechanical crashing.

carbonation and template removing. During carbonization of PDA without removing the template, $\mathrm{N}$-doped carbons might be contaminated by the templating materials under such high temperature. Direct carbonization of PDA capsules might avoid such issues and simplify the preparation procedure. However, such method has barely been explored due to the fragility of the PDA capsules. After obtaining the ellipsoidal PDA capsules with good mechanical rigidity, we speculate that such PDA capsules can be exploited as the precursor for the carbon capsules with an ellipsoids shape.

Carbonization was performed by heating the ellipsoidal PDA capsule at $800{ }^{\circ} \mathrm{C}$ under $\mathrm{N}_{2}$ atmosphere. As expected, the PDA capsules from one cycle of PDA coating broke into pieces after carbonation and intact carbon capsules barely exist (Fig. 6A and C). In contrast, the PDA capsules from four cycles of PDA coating survived the carbonation conditions, giving rise to intact carbon capsules with a well-reserved ellipsoidal shape (Fig. 6C and D). Three peaks corresponding to C 1s, N 1s and O $1 \mathrm{~s}$ in the XPS analysis confirmed the high purity of N-doped carbon without other undesirable compositions (Fig. 7). ${ }^{30,33}$
Fitting of the high-resolution XPS spectrum of $\mathrm{N}$ 1s leads to four sub-peaks that corresponds to different electronic states of the nitrogen element in the carbon capsules: pyridinic $\mathrm{N}(398.51$ $\mathrm{eV})$, pyrrolic $\mathrm{N}(400.60 \mathrm{eV})$, graphitic $\mathrm{N}(401.30 \mathrm{eV})$, and pyridine N-oxide (N-X, $402.89 \mathrm{eV}$ ) (Fig. 7B). The two peaks at ca. 1350 and $1590 \mathrm{~cm}^{-1}$ in the Raman spectrum can be assigned to the $\mathrm{D}$ and $\mathrm{G}$ band, respectively. The $\mathrm{D}$ band is correlated with the disordered carbon and $\mathrm{G}$ band the graphitic carbon. The ratio of the intensity of the $\mathrm{D}$ to $\mathrm{G}$ band is $c a$. 0.82 , indicating the graphitic nature of the $\mathrm{N}$-doped carbon capsules. ${ }^{31}$ These results are consistent with previous works that also use polydopamine as the carbon precursor. ${ }^{30,31,33,34}$

\subsection{Rigid ellipsoidal PDA capsules as effective absorbents for dyes in contaminated water}

In the above section, we have demonstrated that the ellipsoidal PDA capsules obtained from four cycles of iterating PDA coating can be turned into ellipsoidal N-doped carbon capsules. Hereafter, we further demonstrate the application of the rigid PDA capsules as effective absorbents to purify dye-contaminated 

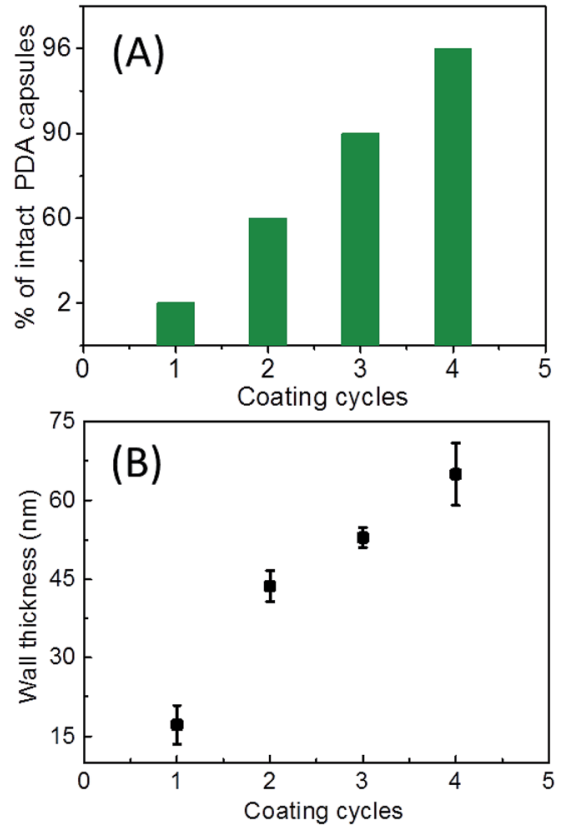

Fig. 5 Percentage of intact ellipsoidal capsules (A) and the thickness of the PDA shell (B) versus the number of the coating cycle.

water. Several previous works have confirmed that PDA based materials are excellent absorbents of several metal ions and some dyes. ${ }^{44,57,58}$ Especially, Quan Xu and coauthors have found that pure PDA microspheres can effectively remove methylene blue $(\mathrm{MB})$ from water and achieve an adsorption capacity of $90.7 \mathrm{mg} \mathrm{g}^{-1}$ at equilibrium at $25^{\circ} \mathrm{C}$, which is among the highest of several kinds of materials. ${ }^{44}$ With the large hollow, nanoscale shell and anisotropic shape, our current PDA capsules are expected to have larger specific surface area that might lead to enhanced adsorption efficiency of organic dyes.

Using MB as the model compound, we performed the adsorption experiments under the identical conditions of the PDA microspheres used by Quan Xu's group, in order to compare with their results. ${ }^{44}$ The time-dependent adsorption capacity was presented in Fig. 8A. With the current ellipsoidal PDA capsules, an adsorption capacity of $200 \mathrm{mg} \mathrm{g}^{-1}$ was achieved during two hours, which is much higher than $90.7 \mathrm{mg} \mathrm{g}^{-1}$ of the pure PDA microspheres under the same conditions and is comparable with that of the chemically modified mixed hardwoods $\left(237.4 \mathrm{mg} \mathrm{g}^{-1}\right.$ ) (Table S1 in ESI $\dagger$ ). ${ }^{59}$ The mechanism of the excellent adsorption capability of PDA based materials for MB is attributed to the $\pi-\pi$ stacking and electrostatic interactions. ${ }^{44}$ Other physical parameter should play critical roles in such dramatic difference between hollow PDA capsules and solid microspheres. To confirm this assumption, the specific area of PDA capsules was determined by the nitrogen adsorption-desorption isotherm at $77 \mathrm{~K}$ (Fig. 8B). The BET surface area of PDA capsules calculated by the standard Brunauer-EmmettTeller (BET) method is $300 \mathrm{~m}^{2} \mathrm{~g}^{-1}$ while the solid PDA microspheres only have a BET of $13.77 \mathrm{~m}^{2} \mathrm{~g}^{-1}$. Such difference is consistent with the general rules that hollow particles have a higher surface-to-volume ratio than the solid counterpart. Both the external and interior surfaces of the PDA capsules are available for interactions with MB. In addition, the interior cavity can further shorten mass transport distance. These factors together contribute to the impressive adsorption capacity of PDA capsules.
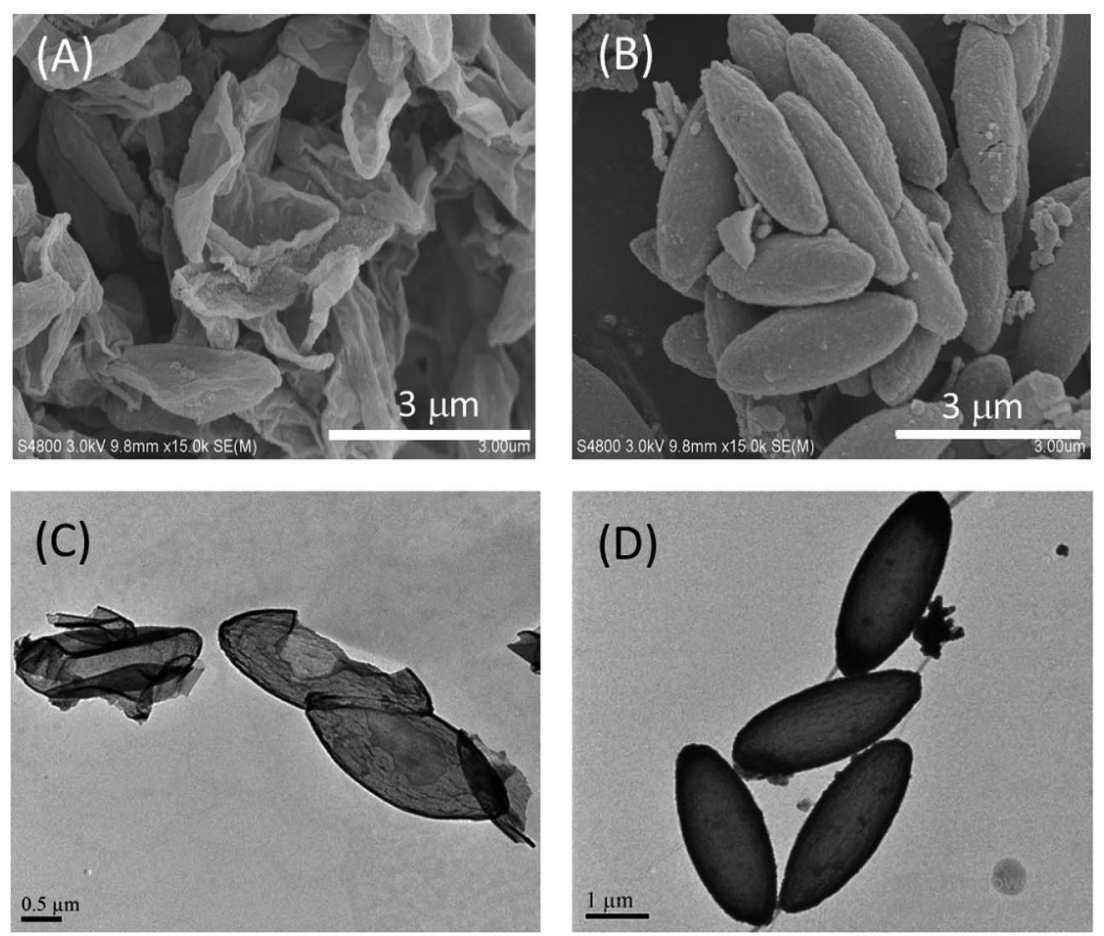

Fig. 6 SEM and TEM of N-doped carbon capsules with an ellipsoidal shape via carbonation of PDA capsules obtained from one time (A and C) and four-time of ( $C$ and D) PDA coating. 

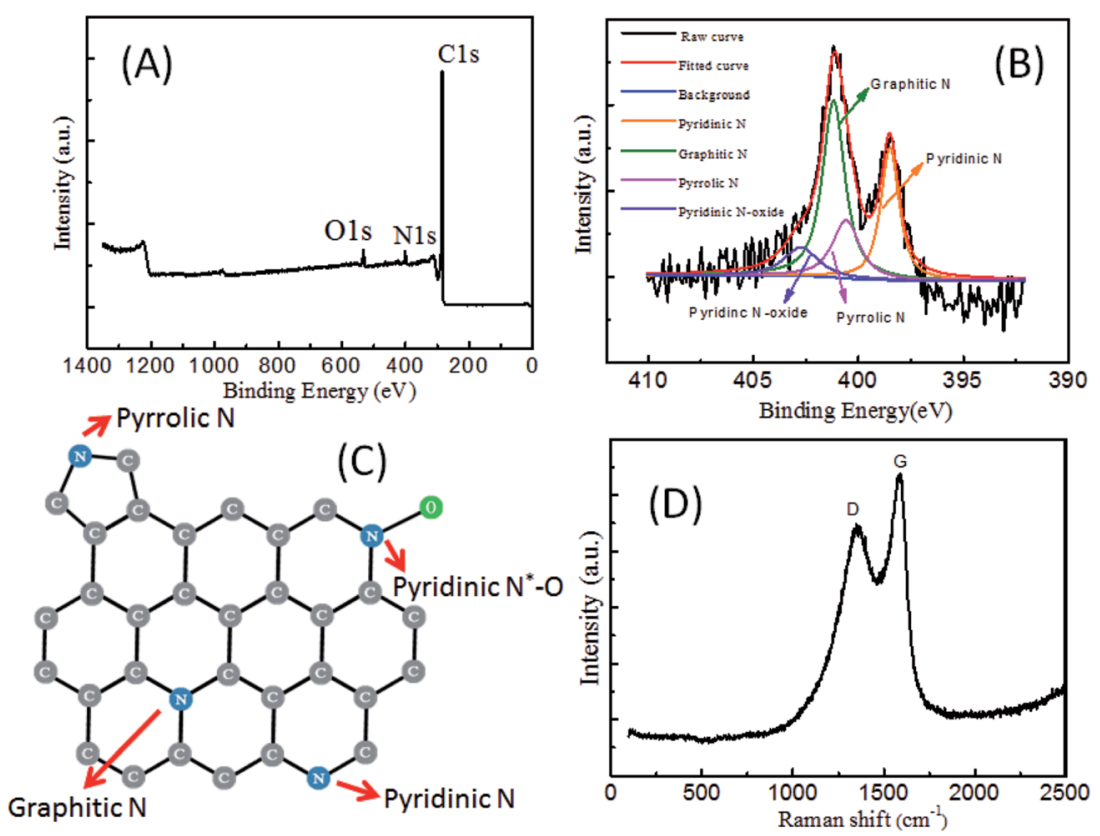

Fig. 7 XPS and Raman spectrum of N-doped carbon capsules with an ellipsoidal shape. The carbon capsules are those presented in Fig. 6C and D. (A) Survey of XPS spectrum. (B) High-resolution XPS spectrum of N 1s and fitting. (C) Chemical structure of the N-doped carbon based on the fitting of (B). (D) Raman spectrum with the two peaks assigned to the D- and G-band.
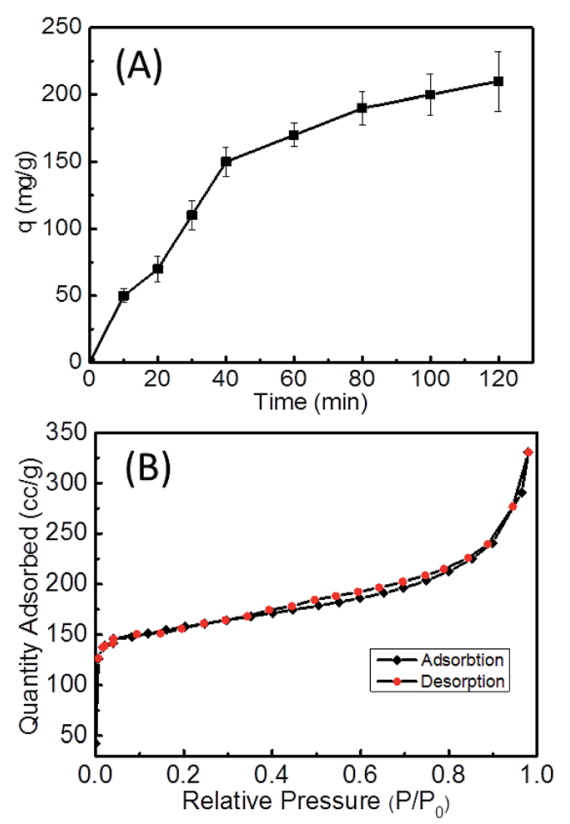

Fig. 8 Time-dependent adsorption efficiency for methylene blue (MB) (A) and nitrogen adsorption-desorption isotherm at $77 \mathrm{~K}(\mathrm{~B})$ of PDA capsules.

Compared to their spherical counterparts, capsules with an ellipsoidal or other non-spherical shape have many additional advantages such as enhanced cargo loading efficiency for drug delivering, improved cell uptake in the cases of cancer treatment and enhanced packing efficiency in solid materials as indicated by previous works. ${ }^{13-15}$ Relative to the current work, we think the advantage of enhanced packing efficiency in solid materials is very beneficial. In the format of dried powders in which particles are randomly packed, ellipsoidal particles can randomly pack more densely and achieve a volume fraction $(\varphi)$ up to 0.74 , higher than 0.64 of spherical ones. ${ }^{13}$ The higher number of degrees of freedom per particle and thus the larger number of particle contacts required to mechanically stabilize the packing are responsible for the higher density of random packing of ellipsoids. ${ }^{13}$ When the dried ellipsoidal PDA capsules in the form of powders are packed into columns for adsorption of MB from water, their high packing efficiency can lead to higher mass/volume ratio. In another words, if the column volume is the same, more ellipsoidal PDA capsules can be integrated into the same column than the spherical capsules (if both have the same morphological volume). Dense packing also means there is less void volume which also benefits the MB adsorption. Due to the same reason, ellipsoidal carbon capsules might achieve better electrochemical performance when being used as electrolyte materials..$^{15}$

\section{Conclusions}

In the current work, the morphology and mechanical rigidity of polydopamine (PDA) capsules were addressed simultaneously. PDA derived from oxidation induced self-polymerization of dopamine is an excellent coating material that can coat nonspherical ellipsoidal polystyrene particles with a PDA layer, regardless of the size and aspect ratio. Dissolving the PS template by THF produced ellipsoidal PDA capsules that accurately replicate the details of the templates such as aspect ratios, high curvature at the tips of the particles, etc. The ellipsoidal PDA capsules buckle, collapse following the way pre-determined by 
the anisotropic surface distribution of the stress produced by airdrying. Iterating coating the PDA coated PS ellipsoids is an effective way to increase the thickness of the PDA shell and to enhance the mechanical rigidity of the PDA capsules, without producing side-products of pure PDA. Compared to PDA capsules from one-time PDA coating, the one from four-time coating can survive carbonization at temperature as high as $800{ }^{\circ} \mathrm{C}$, producing $\mathrm{N}$-doped carbon capsules with an ellipsoidal shape that might be used for catalysis and energy materials. Furthermore, the rigid PDA capsules can work as a high-efficiency adsorbent for removing cationic dyes such as methylene blue from aqueous solution. An adsorption efficiency as high as $200 \mathrm{mg} \mathrm{g}^{-1}$ was achieved. These materials can be explored to purify water contamination caused by dye industries.

\section{Acknowledgements}

This work was supported by the National Natural Science Foundation of China (No. 21274067), the Fundamental Research Funds for the Central Universities and PCSIRT (IRT1257).

\section{Notes and references}

1 H. Lee, S. M. Dellatore, W. M. Miller and P. B. Messersmith, Science, 2007, 318, 426-430.

2 Y. Liu, K. Ai and L. Lu, Chem. Rev., 2014, 114, 5057-5115.

3 X. Liu, J. Cao, H. Li, J. Li, Q. Jin, K. Ren and J. Ji, ACS Nano, 2013, 7, 9384-9395.

4 H. Li, Y. Jia, X. Feng and J. Li, J. Colloid Interface Sci., 2017, 487, 12-19.

5 J. Cui, Y. Yan, G. K. Such, K. Liang, C. J. Ochs, A. Postma and F. Caruso, Biomacromolecules, 2012, 13, 2225-2228.

6 B. Yu, D. A. Wang, Q. Ye, F. Zhou and W. Liu, Chem. Commun., 2009, 6789-6791.

7 A. Postma, Y. Yan, Y. Wang, A. N. Zelikin, E. Tjipto and F. Caruso, Chem. Mater., 2009, 21, 3042-3044.

8 X. Wang, J. Feng, Y. Bai, Q. Zhang and Y. Yin, Chem. Rev., 2016, 116, 10983-11060.

9 J. Cui, Y. Wang, A. Postma, J. Hao, L. Hosta-Rigau and F. Caruso, Adv. Funct. Mater., 2010, 20, 1625-1631.

10 H. Xu, X. Liu and D. Wang, Chem. Mater., 2011, 23, 51055110.

11 D. Park, J. Kim, Y. M. Lee, J. Park and W. J. Kim, Adv. Healthcare Mater., 2016, 5, 2019-2024.

12 Y. Zhai, F. Ishizuka, M. H. Stenzel, A. M. Granville and P. B. Zetterlund, J. Polym. Sci., Part A: Polym. Chem., 2017, 55, 365-370.

13 A. Donev, I. Cisse, D. Sachs, E. A. Variano, F. H. Stillinger, R. Connelly, S. Torquato and P. M. Chaikin, Science, 2004, 303, 990-993.

14 X. Li, M. Bao, Y. Weng, K. Yang, W. Zhang and G. Chen, J. Mater. Chem. B, 2014, 2, 5569-5575.

15 Z. Lin, H. Tian, F. Xu, X. Yang, Y. Mai and X. Feng, Polym. Chem., 2016, 7, 2092-2098.

16 O. Shchepelina, V. Kozlovskaya, S. Singamaneni, E. Kharlampieva and V. V. Tsukruk, J. Mater. Chem., 2010, 20, 6587-6603.
17 X. W. D. Lou, L. A. Archer and Z. Yang, Adv. Mater., 2008, 20, 3987-4019.

18 O. Shchepelina, V. Kozlovskaya, E. Kharlampieva, W. Mao, A. Alexeev and V. V. Tsukruk, Macromol. Rapid Commun., 2010, 31, 2041-2046.

19 M. Sindoro and S. Granick, J. Am. Chem. Soc., 2014, 136, 13471-13473.

20 J. Xue, W. Zheng, L. Wang and Z. Jin, ACS Biomater. Sci. Eng., 2016, 2, 489-493.

21 M.-L. Zhu, Y.-L. Li, Z.-M. Zhang and Y. Jiang, $R S C A d v ., ~ 2015$, 5, 33262-33268.

22 L. Zhang, J. Shi, Z. Jiang, Y. Jiang, S. Qiao, J. Li, R. Wang, R. Meng, Y. Zhu and Y. Zheng, Green Chem., 2011, 13, 300306.

23 M. Kohri, H. Kohma, Y. Shinoda, M. Yamauchi, S. Yagai, T. Kojima, T. Taniguchi and K. Kishikawa, Polym. Chem., 2013, 4, 2696-2702.

24 M. A. Rahim, H. Ejima, K. L. Cho, K. Kempe, M. Müllner, J. P. Best and F. Caruso, Chem. Mater., 2014, 26, 1645-1653.

25 F. Wang, J. Feng, W. Tong and C. Gao, J. Mater. Chem., 2007, 17, 670-676.

26 J. Han, G. Xu, B. Ding, J. Pan, H. Dou and D. R. MacFarlane, J. Mater. Chem. A, 2014, 2, 5352-5357.

27 Y. Xie, L. Fang, H. Cheng, C. Hu, H. Zhao, J. Xu, J. Fang, X. Lu and J. Zhang, J. Mater. Chem. A, 2016, 4, 15612-15620.

$28 \mathrm{X} . \mathrm{Xu}$ and S. A. Asher, J. Am. Chem. Soc., 2004, 126, 79407945.

29 H. Jin, C. L. Mangun, A. S. Griffin, J. S. Moore, N. R. Sottos and S. R. White, Adv. Mater., 2014, 26, 282-287.

30 R. Liu, S. M. Mahurin, C. Li, R. R. Unocic, J. C. Idrobo, H. Gao, S. J. Pennycook and S. Dai, Angew. Chem., Int. Ed., 2011, 50, 6799-6802.

31 Z.-C. Yang, Y. Zhang, J.-H. Kong, S. Y. Wong, X. Li and J. Wang, Chem. Mater., 2013, 25, 704-710.

32 R.-L. Liu, Y. Liu, X.-Y. Zhou, Z.-Q. Zhang, J. Zhang and F.-Q. Dang, Bioresour. Technol., 2014, 154, 138-147.

33 L. Zhang, S. Roy, Y. Chen, E. K. Chua, K. Y. See, X. Hu and M. Liu, ACS Appl. Mater. Interfaces, 2014, 6, 18644-18652.

34 C. Liu, J. Wang, J. Li, R. Luo, J. Shen, X. Sun, W. Han and L. Wang, ACS Appl. Mater. Interfaces, 2015, 7, 18609-18617.

35 H. Ejima, N. Yanai, J. P. Best, M. Sindoro, S. Granick and F. Caruso, Adv. Mater., 2013, 25, 5767-5771.

36 S. Lin, C.-T. Chen, I. Bdikin, V. Ball, J. Grácio and M. J. Buehler, Soft Matter, 2014, 10, 457-464.

37 J. Shi, C. Yang, S. Zhang, X. Wang, Z. Jiang, W. Zhang, X. Song, Q. Ai and C. Tian, ACS Appl. Mater. Interfaces, 2013, 5, 9991-9997.

38 X. Chen, Y. Yan, M. Müllner, M. P. van Koeverden, K. F. Noi, W. Zhu and F. Caruso, Langmuir, 2014, 30, 2921-2925.

39 M. Zheng, Y. Liu, S. Zhao, W. He, Y. Xiao and D. Yuan, Inorg. Chem., 2010, 49, 8674-8683.

40 J. Pan, Y. Ma, J. Zeng, X. Niu, T. Zhang, F. Qiu, Y. Peng and Y. Yan, Chem. Eng. J., 2016, 302, 670-681.

41 M. Lee, J. Rho, D. E. Lee, S. Hong, S. J. Choi, P. B. Messersmith and H. Lee, ChemPlusChem, 2012, 77, 987-990. 
42 C. Li, Z.-J. Qian, C. Zhou, W. Su, P. Hong, S. Liu, L. He, Z. Chen and H. Ji, RSC Adv., 2014, 4, 47848-47852.

43 P. Zhang, L. Yang, Q. Li, S. Wu, S. Jia, Z. Li, Z. Zhang and L. Shi, ACS Appl. Mater. Interfaces, 2017, 9, 7648-7657.

44 J. Fu, Z. Chen, M. Wang, S. Liu, J. Zhang, J. Zhang, R. Han and Q. Xu, Chem. Eng. J., 2015, 259, 53-61.

45 W. Wang, Y. Jiang, S. Wen, L. Liu and L. Zhang, J. Colloid Interface Sci., 2012, 368, 241-249.

46 R. A. Zangmeister, T. A. Morris and M. J. Tarlov, Langmuir, 2013, 29, 8619-8628.

47 Y. Cong, T. Xia, M. Zou, Z. Li, B. Peng, D. Guo and Z. Deng, J. Mater. Chem. B, 2014, 2, 3450-3461.

48 C. Ho, A. Keller, J. Odell and R. Ottewill, Colloid Polym. Sci., 1993, 271, 469-479.

49 Y. Lu, Y. Yin and Y. Xia, Adv. Mater., 2001, 13, 271-274.

50 J. R. Liebscher, R. Mrówczyński, H. A. Scheidt, C. Filip, N. D. Hădade, R. Turcu, A. Bende and S. Beck, Langmuir, 2013, 29, 10539-10548.
51 S. Wang, X. Zhao, S. Wang, J. Qian and S. He, ACS Appl. Mater. Interfaces, 2016, 8, 24368-24384.

52 S. Kang, M. Baginska, S. R. White and N. R. Sottos, ACS Appl. Mater. Interfaces, 2015, 7, 10952-10956.

53 Q. Liu, B. Yu, W. Ye and F. Zhou, Macromol. Biosci., 2011, 11, 1227-1234.

54 C. Xu, Q. Wang, H. Xu, S. Xie and Z. Yang, Colloid Polym. Sci., 2007, 285, 1471-1478.

55 X. Liang, V. Kozlovskaya, Y. Chen, O. Zavgorodnya and E. Kharlampieva, Chem. Mater., 2012, 24, 3707-3719.

56 A. Lazarus, H. Florijn and P. Reis, Phys. Rev. Lett., 2012, 109, 144301.

57 Y. Yu, J. G. Shapter, R. Popelka-Filcoff, J. W. Bennett and A. V. Ellis, J. Hazard. Mater., 2014, 273, 174-182.

58 S. Du, Z. Liao, Z. Qin, F. Zuo and X. Li, Catal. Commun., 2015, 72, 86-90.

59 Y.-Z. Zhang, Y.-Q. Jin, Q.-F. Lü and X.-S. Cheng, Ind. Eng. Chem. Res., 2014, 53, 4247-4253. 